\title{
Repeated production of fatty acid methyl ester with activated bleaching earth in solvent-free system
}

\author{
Dongning Du, ${ }^{1}$ Masayasu Sato, ${ }^{2}$ Masahiro Mori, ${ }^{1}$ Enoch Y. Park ${ }^{1, *}$
}

Laboratory of Biotechnology, Department of Applied Biological Chemistry, Faculty of Agriculture, Shizuoka University, 836 Ohya, Suruga-ku, Shizuoka 422-8529, ${ }^{1}$

Intellectual Property Department, Mizusawa Industrial Chemicals Ltd., 1-13-6

Nihonbashimuromachi, Chuo-ku, Tokyo 103-0022, Japan ${ }^{2}$

Received 2005; accepted 2005

\footnotetext{
* Corresponding author. Phone/fax: +81-54-238-4887.

E-mail address: yspark@agr.shizuoka.ac.jp (E.Y. Park).
} 


\begin{abstract}
The enzymatic transesterification of rapeseed oil and methanol with activated bleaching earth (ABE) as a medium was investigated in a solvent-free system. The initial fatty acid methyl ester (FAME) production rate in the solvent-free system was 2.3-fold lower than that in the organic solvent system, but there was not much difference between the overall reaction rates in the solvent and solvent-free systems. When $\mathrm{ABE}$ was added to the reaction mixture consisting of rapeseed oil, methanol, and lipase, the repeated batch production of FAME was conducted for 9 cycles without a significant inactivation of lipase. However, it was only repeated 6 times in the rapeseed oil-fed batch process, because of the inactivation of lipase. The FAME yield based on used lipase in the repeated batch process was 55.8 grams FAME per grams used lipase, which was two-fold higher than that of the oil-fed batch process.
\end{abstract}

Keywords: Activated bleaching earth; Lipase; Solvent free; Fatty acid methyl ester; Esterification 


\section{Introduction}

As the source of petroleum becomes instable and the world's petroleum reserves become depleted, much research on the use of renewable resources as energy sources has been carried out $[1,2]$. The substitution of fatty acid methyl ester (FAME) for a part of diesel fuel is one method for reducing petroleum consumption [3]. FAME, which is collectively known as biodiesel, can be produced from animal fats and vegetable oil by transesterification of triglycerides with methanol [4].

Enzymatic hydrolysis for the production of FAME has an advantage compared with the chemical method [5]. However, there are two major problems in using enzymatic hydrolysis. First, lipase is expensive and it is liable to be deactivated by a lower alcohol [6, 7]. To overcome this problem, some researchers carried out the reaction in an organic solvent to decrease the methanol concentration to improve lipase activity [8]. However, because organic solvents are very harmful to health and the environment, there may be safety issues regarding this process. For scale-up and continuous production of FAME in industry, the reaction without the use of organic solvent is an appropriate approach. Second, a heterogeneous reaction occurs because of the immiscibility of methanol and triglyceride, which causes a negative effect on the production of FAME [9].

We utilized activated bleaching earth (ABE) as a medium for the production of FAME $[10,11]$. ABE is a good adsorbent used in the oil refining industry due to its high adsorption capacity of coloring materials. After upgrading crude oils in the oil refining process, the waste ABE contains nearly $40 \%$ of its weight as waste oil and is discarded as a waste material. Therefore, the reutilization of waste ABE from this source is a good alternative for 
biodiesel production and it may make the biodiesel price competitive in the market. When vegetable oil extracted from this waste $\mathrm{ABE}$ was used for esterification using Candida cylindracea lipase, the conversion was only $13 \%(w / w)[11]$. However, with the presence of $\mathrm{ABE}$ in the reaction mixture, the conversion improved dramatically, to higher than $80 \%$ for an $8 \mathrm{~h}$ reaction [11], which it makes possible for the waste $\mathrm{ABE}$ to be used in the production of FAME without any pretreatment. In the transesterification reaction, ABE adsorbs methanol, which lessens the inactivation of lipase by methanol and maintains a high reaction rate [12]. Additionally, after transesterification, the waste $\mathrm{ABE}$ that contains lipase can be regarded as immobilized lipase that can be reused in the next cycle reaction, which will reduce the catalyst cost.

For the reutilization of ABE, we examined the repeated production of FAME using $\mathrm{ABE}$ as a reaction medium without any organic solvent. Two processes containing ABE were considered for the repeated FAME production, and lipase inactivation and the productivity the FAME in each process is discussed.

\section{Materials and Methods}

\subsection{Materials}

Lipases powders (QLM) from Alcaligenes. sp were purchased from Meito Sangyo (Nagoya, Japan). The methanol and rapeseed oil used for methanolysis were analytical grade and were purchased from Wako Pure Chemical Industries Ltd. (Osaka, Japan). All other chemicals used were of analytical reagent grade. Mizusawa Industrial Chemicals Ltd. (Tokyo, Japan) provided waste ABE. 


\subsection{Methanolysis of rapeseed oil}

The initial conditions were adopted from a previous study [3]. The reaction mixtures consisted of 2 g rapeseed oil, $3 \mathrm{~g}$ ABE, and $0.06 \mathrm{~g}$ lipase (3\% (w/w based on oil weight)) under the conditions of $37^{\circ} \mathrm{C}$ and 200 rounds per minute (rpm) in a reciprocal shaker (Bio Shaker, Takasaki Scientific Instruments Co., Japan). The molar ratio of oil to methanol was varied from 1:1 to $1: 8$.

To study the effect of ABE in the solvent-free system, the initial FAME production rate was investigated under a lipase concentration of $10 \%$, on the basis of $\mathrm{ABE}$ and rapeseed oil weight. Because the reaction conditions, including ABE, lipase, methanol, and rapeseed oil concentrations, are known at time zero, the initial FAME production rate and the specific FAME production rate were defined as,

$$
\begin{aligned}
& \text { Initial FAME production rate }(\mathrm{mM} / \mathrm{min})=\left[\frac{d P}{d t}\right]_{t=0} \\
& \text { Specific FAME production rate }(\mathrm{mmole} / \mathrm{min} / \mathrm{g} \text { lipase })=\frac{\left[\frac{d P}{d t}\right]_{t=0}}{C_{0}}
\end{aligned}
$$

where $P, C_{0}$, and $t$ denote concentrations of produced FAME and used lipase, and reaction time, respectively.

\subsection{Repeated production of FAME from rapeseed oil in reactor}

Two kinds of repeated production of FAME were performed. One was a repeated batch 
process in which the produced FAME was separated from the reaction mixture and then new rapeseed oil and methanol were added to the reactor for the next cycle reaction. The other is a rapeseed oil-fed batch process. In every cycle reaction, rapeseed oil and methanol were added at regular intervals for the production of FAME. The produced FAME was harvested at the final cycle reaction. The starting reactants consisted of $234 \mathrm{~g}$ of ABE, 156 g of rapeseed oil, $23 \mathrm{~g}$ of methanol (the molar ratio of methanol and rapeseed oil 4:1), and $39 \mathrm{~g}$ of lipase, respectively. The reaction was carried out in a 2-L reactor mixing at $200 \mathrm{rpm}$ using a stirrer (RZR 2020, Heidolph, Kelheim, Germany) and at a reaction temperature of $37^{\circ} \mathrm{C}$. For the second cycle, rapeseed oil (156 g) and methanol (23 g) were added to the reactor, and the reaction was repeated under the same reaction conditions as the first cycle.

\subsection{Analytical methods}

Lipase activity (IU/ml), defined as the amount of lipase that liberated $1 \mu \mathrm{l}$ of free thiol groups per minute, was measured using a Lipase Kit S (Dainippon Pharmaceutical, Osaka). Glycerol was measured using Kit F (R-Biopharm AG, Darmstadt, Germany). Five hundred microliters of the sample after methanolysis was extracted and centrifuged at $7155 \mathrm{~g}$ in a microcentrifuge. A 25- $\mu$ g aliquot of supernatant was dissolved in $1.5 \mathrm{~g}$ of chloroform, and 2- $\mu \mathrm{l}$ aliquot of the solution was injected onto a gas chromatograph (GC-14B, Shimadzu, Kyoto) coupled with a glass column (3 mm x $2 \mathrm{~mm}$ ) packed with 5\% Advans DS on 80/100 meshes Chromosorb W (Shimadzu, Kyoto) and a flame ionized detector. Nitrogen gas as a carrier flowed at a rate of $50 \mathrm{ml} / \mathrm{min}$, and the pressure of the used hydrogen gas and air were 0.6 and $0.5 \mathrm{~kg} / \mathrm{cm}^{2}$, respectively. The detector and injection port temperatures were $240^{\circ} \mathrm{C}$, and an isothermal column temperature was maintained at $190^{\circ} \mathrm{C}$. All GC 
measurements were performed in triplicate and the concentration of FAME was determined from the methyl ester fatty acids of rapeseed oil, utilizing methyl pentadecanoate as an internal standard.

\section{Result and discussion}

\subsection{Effect of ratio of rapeseed oil and methanol on FAME production}

The crystal lattice of ABE is a three-layered structure, namely, silica-alumina-silica, which confers a large specific surface area and large adsorption capacity because of its porous structure. Rapeseed oil and methanol in liquid phase were immediately adsorbed onto the ABE generating a rapeseed-oil-methanol-ABE complex. Therefore, the balance of methanol and rapeseed oil is important in solvent-free methanolysis. To optimize the molar ratio of methanol to rapeseed oil, the addition of methanol to the reaction mixture was investigated using molar ratios of methanol to rapeseed oil from 1:1 to 1:8 (Fig. 1). The theoretical molar ratio of methanol to rapeseed oil is $1: 3$. When the ratio was $1: 1$ the FAME conversion was lower than $50 \%$, and when the ratio was $1: 3$ it was $67 \%$. This might be due to a diffusion limitation of methanol in the lipase-catalyzed transesterification on the surface of the ABE. When the molar ratio was 1:4, the FAME conversion reached to $81 \%$ in a reaction time of $4 \mathrm{~h}$. However, in the case of a methanol molar ratio higher than 1:4, the conversion of rapeseed oil to FAME decreased and was lower than 60\%. The low conversion efficiency using a high molar ratio of methanol is caused by methanol inhibition of the catalytic reaction, which was shown elsewhere [11]. 
3.2. Comparison of FAME production rate between solvent and solvent-free system

The FAME production rate in a solvent-free system was compared to that using $n$-hexane as an organic solvent. When an organic solvent was used in the enzymatic esterification, the dependence of lipase concentration was $3.7 \mathrm{mmole} / \mathrm{min} / \mathrm{g}$ used lipase, whereas in a solvent-free system it was $1.6 \mathrm{mmole} / \mathrm{min} / \mathrm{g}$ used lipase (Fig. 2A). The specific FAME production rate in the organic solvent system was 2.3-fold higher than that of the solvent-free system.

However, the overall FAME conversion, reached to 90\%; thus, there was not much difference between the solvent and the solvent-free system (Fig. 2B). When the lipase concentration was $10 \%(\mathrm{w} / \mathrm{w})$ in the organic solvent system, the FAME production rate was $495 \mathrm{mM} / \mathrm{min}$ during the first hour of the reaction, $87 \mathrm{mM} / \mathrm{min}$ between 1 and $4 \mathrm{~h}$, and 32 $\mathrm{mM} / \mathrm{min}$ between 4 and $7 \mathrm{~h}$. However, in the case of the solvent-free system, the FAME production rate was $165 \mathrm{mM} / \mathrm{min}$ during the first four hours of the reaction and $74 \mathrm{mM} / \mathrm{min}$ between 4 and $7 \mathrm{~h}$. The initial FAME production rate of the organic solvent system was 2-3 times higher than that of solvent-free system (Fig. 2B). However, in the case of the solvent-free system, the initial FAME production rate was lower than that in the solvent system, but it increased slowly. Finally, there was no difference in the overall FAME production rate. When the lipase concentration was $5 \%(w / w)$, there was no difference in the FAME conversion between solvent and solvent-free systems, except for the initial FAME production rate (Fig. 2B).

\subsection{Repeated and oil-fed batch process for FAME production in solvent-free system}


When the FAME conversion was approximately 100\%, one reaction cycle was over. Produced FAME was recovered by centrifugation of the reaction mixture to remove the ABE. This cyclic reaction was repeated 8 times as shown in Fig. 3. As the embedded rapeseed oil on the $A B E$ was converted to FAME by the enzymatic esterification, the $A B E$ adsorbed soon produced FAME. This is the reason why the reaction began with around 50\% FAME conversion every cycle. This process was repeated till the $8^{\text {th }}$ cycle, maintaining the FAME conversion higher than $95 \%$, but at the $9^{\text {th }}$ cycle the FAME conversion decreased to $90 \%$.

To avoid the need to separate FAME from the reaction mixture every cycle, the substrates, rapeseed oil and methanol, were added at a regular interval. This process was repeated five times (Fig. 3), but the FAME production rate gradually decreased and reached to $85 \%$ at the $5^{\text {th }}$ cycle. The produced FAME remained in the reactor, which might cause an inhibitory effect on the catalytic activity of the lipase during the reaction. At the $1^{\text {st }}$ cycle the $\mathrm{ABE}$ adsorbed rapeseed oil and methanol, and methanol-rapeseed oil-ABE complex is formed, but lipase powder remained as a solid phase. When rapeseed oil embedded in the ABE was converted to FAME, rapeseed oil and methanol were added to the $2^{\text {nd }}$ reaction cycle. Throughout this process the amounts of ABE and lipase remained constant, but the amount of liquid phase was increased with the progress of the FAME oil-fed batch production. This causes to dilute the substrates of lipase in the whole reaction system.

\subsection{Comparison of FAME production between two processes}


FAME production is dependent on lipase activity in the reaction mixture. To investigate the inhibitory effect in the two processes on FAME production, residual lipase activity was measured. The repeated production process, in which the produced FAME was separated from the reaction mixture in every cycle, stabilized the lipase activity for 7 cycles without inactivation, and for the $8^{\text {th }}$ and $9^{\text {th }}$ cycles, the activity decreased to $90 \%$ (Fig. 4A). On the other hand, in a rapeseed oil-fed batch production process without FAME separation, the lipase activity decreased gradually and reached $71 \%$ at the $6^{\text {th }}$ cycle (Fig. $4 \mathrm{~A}$ ). This is due to an inhibitory effect of FAME on lipase activity. When the lipase was saturated by FAME, the lipase inactivation had the first-order kinetics (Fig. 4B). The inactivation constant was $-0.0199 \mathrm{~h}^{-1}$ with a correlation coefficient of 0.99 and the half-life of the enzyme was $34.8 \mathrm{~h}$. Therefore, the decrease in FAME production in the oil-fed batch process is consistent with enzyme inactivation. In the case of ABE medium, glycerol was not detected in FAME, and it was found that the glycerol was adsorbed by the ABE, which may favor the reaction towards product formation. In conventional chemical hydrolysis, additional separation processes, for example, dehydration and the separation of glycerol in the FAME, are required.. This reduces the separation of glycerol from the FAME; thus, ABE medium is suitable for continuous large-scale production.

Two FAME production processes are summarized as shown in Table 1. ABE recovered from both processes was $431 \mathrm{~g}$, because of the adsorption of $200 \mathrm{~g}$ of produced FAME. Although the used amount of ABE and lipase were 234 and 39 g, respectively, the repeated batch process produced $2.2 \mathrm{~kg}$ of FAME, while the oil-fed batch process produced $0.9 \mathrm{~kg}$ of FAME, suggesting the repeated batch process produced 2.4-fold more FAME than the oil-fed batch process. The FAME yield per used lipase was also 2.4 times higher in 
the repeated batch process. In our repeated batch process using the solvent-free system, the lipase, which was in existence only as a powder in the reaction mixture, as was ABE, was reused eight times without significant inactivation. This may be due to a kind of immobilization. Immobilization is one of the desirable technologies to reuse lipase without inactivation. Many kinds of supports have been examined for repeated use of lipase in FAME production. Many of them were inorganic supports, such as silica [13], celite [14], resin [15, 16], and kaolinite [17]. Du et al [16] repeatedly reused immobilized lipase ten times at $30^{\circ} \mathrm{C}$. Immobilized Rhizomucor miehei lipase on celite maintained an activity of $90 \%$, even though it was reused 8 times with a stepwise addition of $1 \mathrm{M}$ equivalent of methanol at $5 \mathrm{~h}$ intervals to avoid lipase inactivation [14].

From the view point of the commercial production of FAME using lipase, the manufacturing cost is still an obstruction, of which lipase composes more than $90 \%$. Fifty-six grams of FAME was produced from 1 gram of immobilized lipase in the presence of ABE (Table 1), which indicates the need for 0.018 grams of lipase per 1 gram of FAME production, corresponding $1.8 \%(\mathrm{w} / \mathrm{w})$ of FAME. This is still too high for the commercial production of FAME. Our estimation surmises that the amount of lipase should be lower than $0.5 \%(\mathrm{w} / \mathrm{w})$ of FAME; thus, there is still requirement to develop technology for reducing and repeatedly using lipase without inactivation.

This study shows the possibility using ABE for the production of FAME in a solvent-free system. However, our final goal is to use waste ABE as a medium of transesterification reaction in a solvent-free system. A study on the improvement of the FAME yield per gram lipase using waste $\mathrm{ABE}$ as a reaction medium in a solvent-free system is still required to realize the industrial production of FAME as a fuel substitution. 


\section{Acknowledgements}

This work was carried out as a research project for utilizing advanced technologies in agriculture, forestry and fisheries of the Ministry of Agriculture, Forestry and Fisheries of Japan. The authors thank Mr. Shigemi Sato of Mizusawa Industrial Chemicals, Ltd., of Japan for advising of experiments and for supplying and handling activated bleaching earth.

\section{References}

[1] Noureddini H, Zhu D. Kinetics of transesterification of soybean oil. JAOCS 1997; 74: 1457-63.

[2] Fukuda H, Kondo A, Noda H. Biodiesel fuel production by transesterification of oils. J Biosci Bioeng 2001; 92: 406-16.

[3] Kojima S, Du D, Sato M, Park EY. Efficient production of fatty acid methyl ester from waste activated bleaching earth using diesel oil as organic solvent. J Biosci Bioeng 2004; 98: 420-24.

[4] Clark SJ,Wangner L, Schrock MD, Piennaar PG. Methyl and ethyl soybean esters as renewable fuels for diesel engines. JAOCS 1984; 61; 1632-38.

[5] Ikwuagwu OE, Ononogbu IC, Njoku OU. Production of biodiesel using rubber [Hevea brasiliensis (Kunth. Muell.)] seed oil. Industrial Crops and Products 2000; 12: 57-62.

[6] Watanabe Y, Samukawa T, Sugihara A, Noda H, Fukuda H, Tominaga Y. Conversion of vegetable oil to biodiesel using immobilized Candida antarctica lipase. J Am Oil Chem Soc 1999; 76:789-93.

[7] Watanabe Y, Shimada Y, Sugihara A, Yominaga A. Conversion of degummed soybean 
oil to biodiesel fuel with immobilized Candida antarctica lipase. J Mol Catal B: Enzym 2002; 17: 151-55.

[8] Kaieda M, Samukawa T, Kondo A, Fukuda H. Effect of methanol and water contents on production of biodiesel fuel from plant oil catalyzed by various lipases in a solvent-free system. J Biosci Bioeng 2001; 91: 12-15.

[9] Violeta M, Egle S, Prutenis J. Solubility of multi-component biodiesel fuel systems. Biores Technol 2005; 96: 611-16.

[10] Lara PV, Park EY. Lipase-catalyzed production of biodiesel fuel from vegetable oils contained in waste activated bleaching earth. Proc Biochem 2003; 38: 1077-82.

[11] Lara PV, Park EY. Potential application of waste activated bleaching earth on the production of fatty acid alkyl esters using Candida cylindracea lipase in organic solvent system. Enz Microb Technol 2004; 34: 270-77.

[12] Mori M, Park EY. Kinetic study of esterification of rapeseed oil contained in waste activated bleaching earth using Candida cylindracea lipase in organic solvent system. J Mol Catal B-Enz, 2005; 37: 95-100.

[13] Hsu A-F, Jones K, Foglia TA, Marmer WN. Immobilized lipase-catalyzed production of alkyl esters of restaurant grease as biodiesel. Biotechnol Appl Biochem, 2002; 36: 181-86.

[14] Soumanou MM, Bornscheuer UT. Improvement in lipase-catalyzed synthesis of fatty acid methyl esters from sunflower oil. Enz Microb Technol, 2003; 33: 97-103.

[15] Murthy MSRC, Basha ZA, Swaminathan T. Hydrolysis of methyloleate using immobilized lipase from Chromobacterium viscosum. Bioproc Eng, 1998; 19: 475-78.

[16] Du W, Xu Y, Liu D. Lipase-catalyzed transesterification of soya bean oil for biodiesel 
production during continuous batch operation. Biotechnol Appl Biochem, 2003; 38: 103-106.

[17] Iso M, Chen B, Eguchi M, Kudo T, Shrestha S. Production of biodiesel fuel from triglycerides and alcohol using immobilized lipase. J Mol Catal B-Enz, 2001; 16: 53-58. 
Table 1

Summaries of FAME production in solvent-free system

\begin{tabular}{rrr}
\hline Process & Repeated batch process & Oil-fed batch process \\
Amount of reactants (g) & 234 & 234 \\
Lipase & 39 & 39 \\
Rapeseed oil & 2028 & 1092 \\
Methanol & 298.2 & 160.3 \\
Final recovered products (g) & & 431 \\
Recovered ABE & 431 & $921.7^{\mathrm{b}}$ \\
FAME & $2176.7^{\mathrm{a}}$ & 23.6 \\
\hline FAME yield (g FAME/g lipase) & 55.8 & \\
\hline
\end{tabular}

${ }^{\mathrm{a}}$ FAME conversion: 98\%; ${ }^{\mathrm{b}}$ FAME conversion: 85\% 


\section{Legends for figures}

Fig. 1. Effect of methanol to rapeseed oil molar ratio on lipase-catalyzed methanolysis of rapeseed oil in solvent-free system. Symbols: 1:1, closed triangles; 1:3, open circles; 1:4, closed circles; 1:6, closed squares; 1:8, open triangles.

Fig. 2. (A) Specific FAME production rate in $n$-hexane (closed circles) and solvent-free system (open circles). (B) Conversion of rapeseed oil to FAME in $n$-hexane (closed symbols) and solvent-free system (open symbols). The reaction was carried out in a 2-L reactor with mixing at $200 \mathrm{rpm}$ and a reaction temperature of $37^{\circ} \mathrm{C}$. Symbols: used lipase concentration was 10\% w/w (circles) and 5\% w/w (triangles).

Fig. 3. Repeated (open circles) and rapeseed oil-fed batch (closed circles) production of FAME in solvent-free system. When the FAME conversion reached $100 \%$ in each cycle, the FAME was separated from the reaction mixture and the next reaction cycle began. Arrows indicate the addition time of reactants. Long and short arrows indicate the addition time of reactants in repeated and oil-fed batch production of FAME, respectively.

Fig. 4. (A) Residual lipase activity of repeated (open circles) and oil-fed batch (closed circles) productions of FAME. (B) Inactivation kinetics of lipase activity in oil-fed batch productions of FAME. Lipase activity was represented as a relative value of the initial one. 
Fig. 1, Du et al

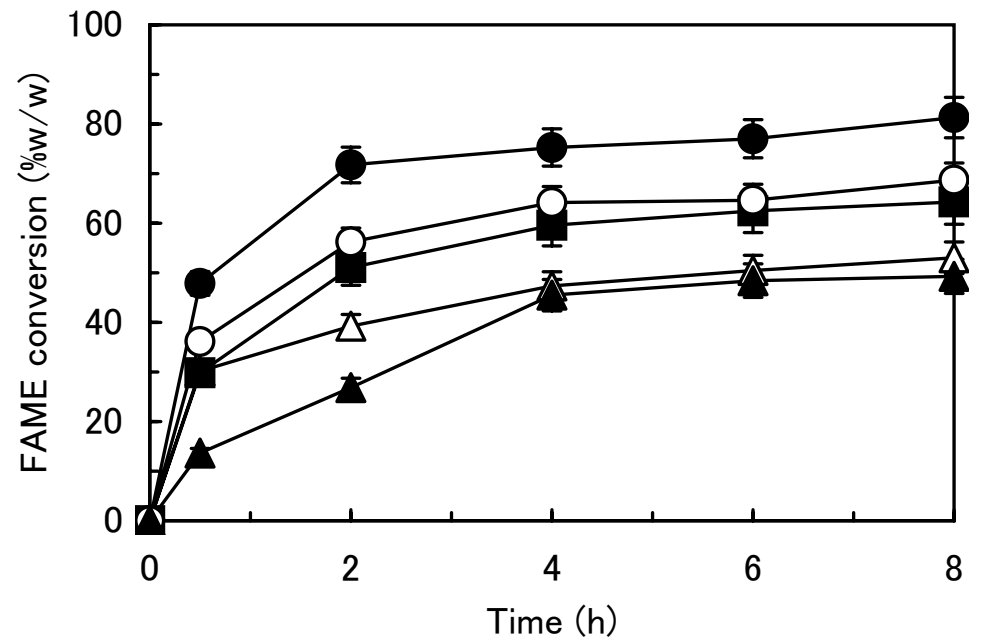


Fig. 2, Du et al.
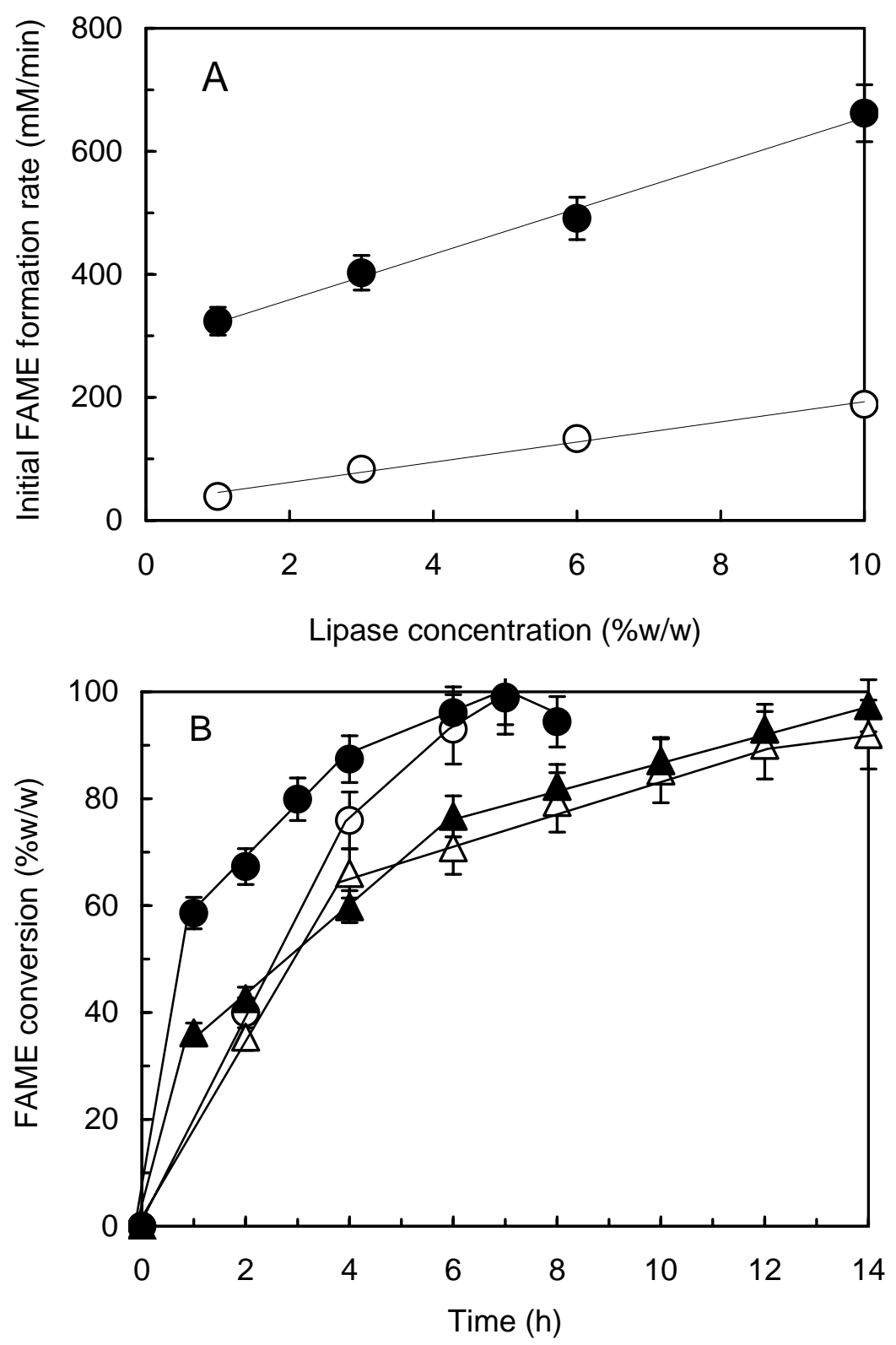
Fig. 3, Du et al.

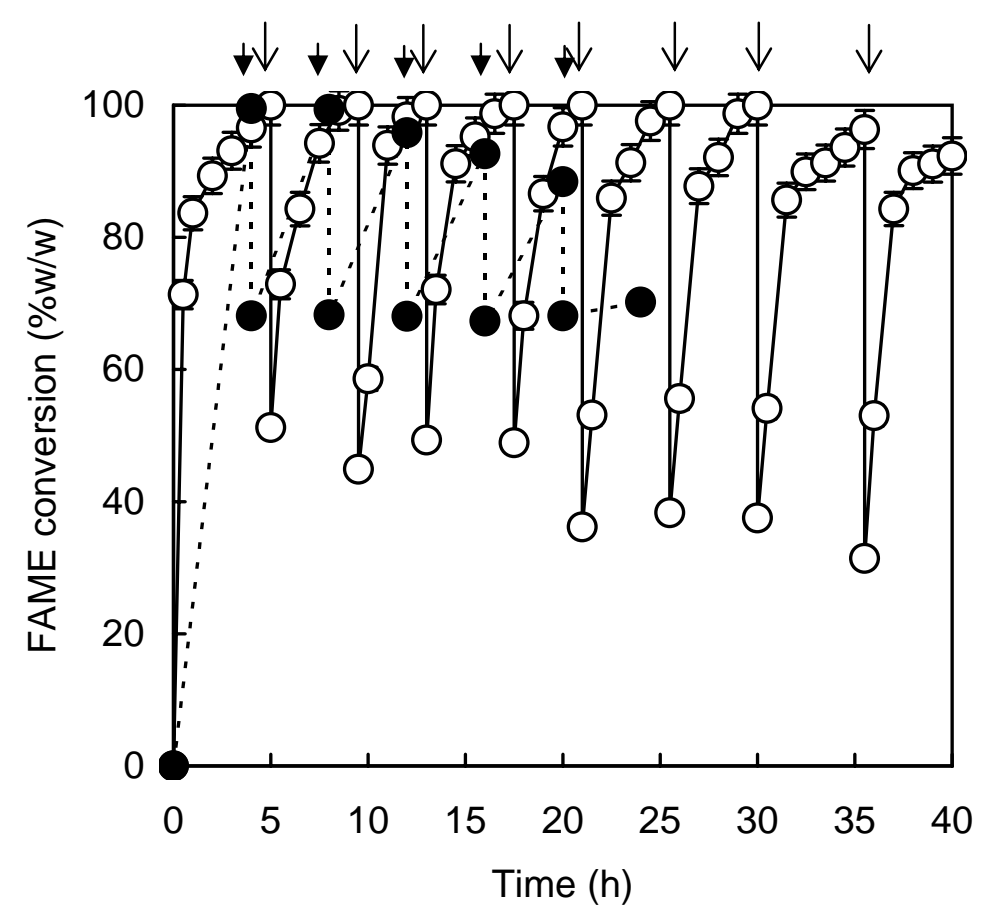



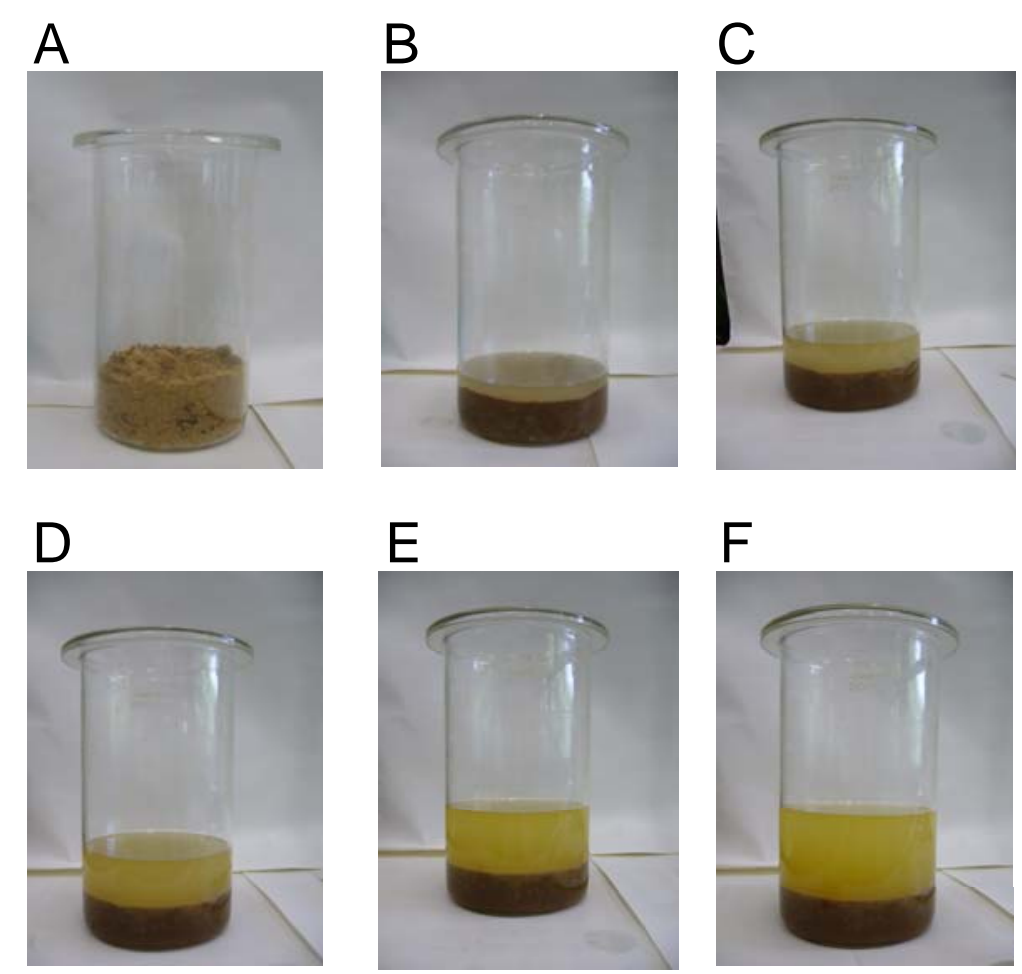
Fig. 5, Du et al.
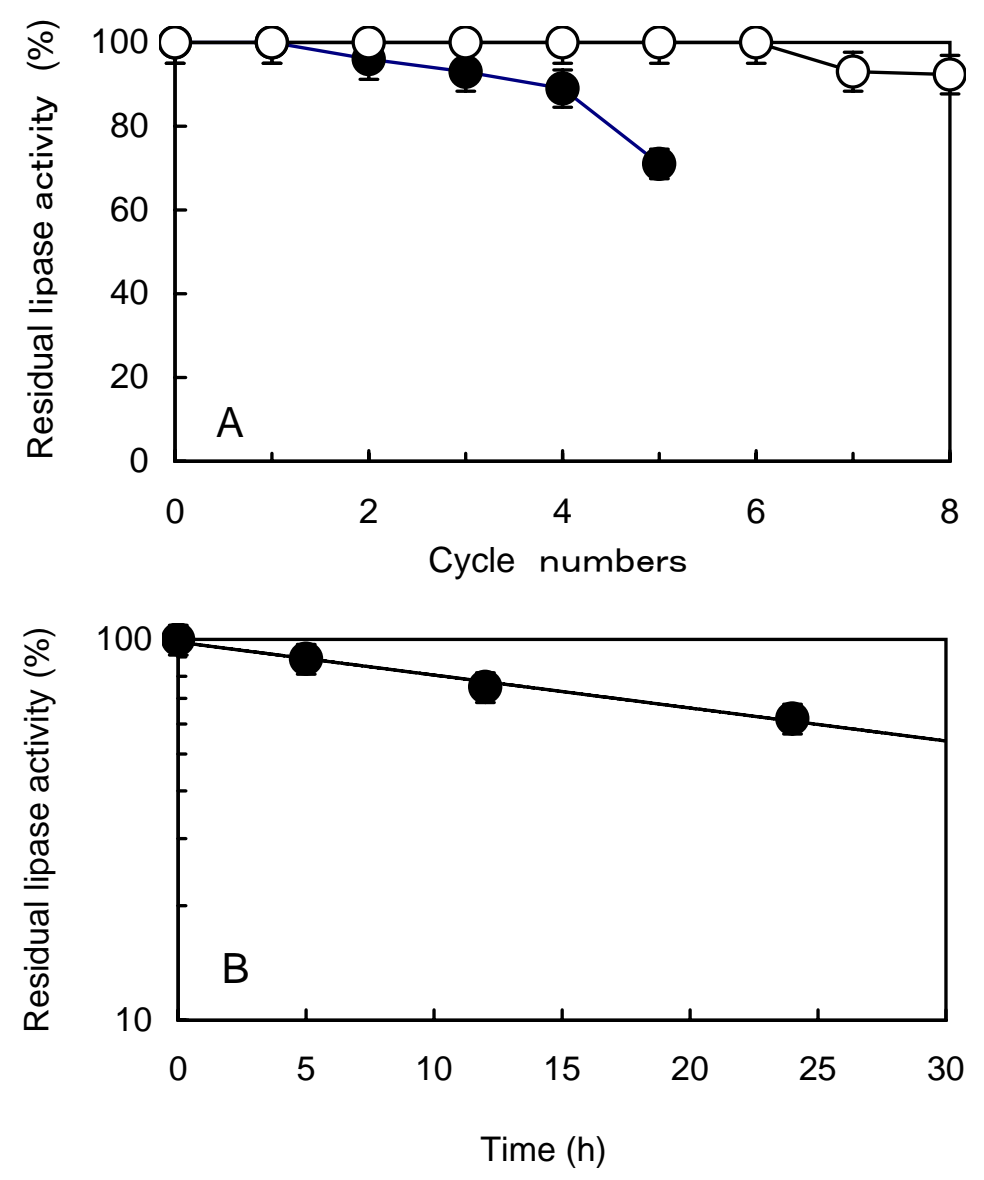